CERN-PH-TH/2006-074

SLAC-PUB-11841

\title{
NNLL QCD Contribution of the Electromagnetic Dipole Operator to $\Gamma\left(\bar{B} \rightarrow X_{s} \gamma\right)$
}

\author{
H.M. Asatrian, A. Hovhannisyan, and V. Poghosyan \\ Yerevan Physics Institute, 375036 Yerevan, Armenia \\ T. Ewerth and C. Greub \\ Inst. for Theoretical Physics, Univ. Berne, CH-3012 Berne, Switzerland \\ T. Hurth ${ }^{1}$ \\ CERN, Dept. of Physics, Theory Unit, CH-1211 Geneva 23, Switzerland \\ SLAC, Stanford University, Stanford, CA 94309, USA
}

\begin{abstract}
We present an independent calculation of that part of the $O\left(\alpha_{s}^{2}\right)$ contribution to the decay width $\Gamma\left(\bar{B} \rightarrow X_{s} \gamma\right)$ which arises from the self-interference term of the electromagnetic dipole operator $\mathcal{O}_{7}$. Using a different method, we find complete agreement with a previous calculation. This NNLL contribution is an important ingredient for the complete NNLL prediction of $\Gamma\left(\bar{B} \rightarrow X_{s} \gamma\right)$ which will resolve the charm quark mass ambiguity appearing at NLL accuracy.
\end{abstract}

${ }^{1}$ Heisenberg Fellow 


\section{Introduction}

Rare $B$ decays as flavor changing neutral currents (FCNCs) are loop-induced processes and highly sensitive probes for new degrees of freedom beyond the SM. But this indirect search for new physics signatures within flavor physics takes place today in complete darkness, given that we have no direct evidence of new particles beyond the SM. However, the day the existence of new degrees of freedom is established by the LHC, specifically when the mass scale of the new physics is fixed, the searches for anomalous phenomena in the flavor sector will become mandatory. In this context, the measurement of theoretically clean rare decays, even when found to be SM-like, will lead to important and valuable information on the structure of the new-physics models and to complementary information to the LHC collider data.

Because physics effects beyond the SM in flavor observables seem to be rather small, as recent experimental data indicate, the focus on theoretically clean observables within the indirect search for new physics is crucial in order to detect specific patterns and to distinguish between various new physics scenarios. Inclusive rare $B$ decays belong to this class of observables because they are dominated by perturbative contributions and nonperturbative effects are generally suppressed by $\Lambda_{\mathrm{QCD}}^{2} / m_{b}^{2}$ within the heavy mass expansion (for a review see [1]).

The inclusive $b \rightarrow s \gamma$ mode is still the most prominent rare decay. The stringent bounds obtained from this mode on various non-standard scenarios (see e.g. [2-9]) indicate the importance of this specific FCNC observable in discriminating new-physics models. It has been measured by several independent experiments [10-14], and the present experimental accuracy is already below the $10 \%$ level. In the HFAG world average of these measurements, a common shape function for the extrapolation to low energies was used; for a photon energy cut $E_{\gamma}>1.6 \mathrm{GeV}$ the average is given by [15]:

$$
\mathrm{BR}\left[\bar{B} \rightarrow X_{s} \gamma\right]=\left(3.55 \pm 0.24_{-0.10}^{+0.09} \pm 0.03\right) \times 10^{-4},
$$

where the errors are combined statistical and systematic, the systematic ones due to shape function, and due to the $d \gamma$ contamination. In the near future, more precise data on this mode are expected from the (upgraded) $B$ factories and from the planned Super- $B$ factories (see [16-18]).

Clearly, the theoretical uncertainty should be reduced accordingly. After completion of the QCD NLL [19-28]. and the electroweak two-loop [29-32] computations, it was generally believed that the theoretical uncertainty of the branching ratio is below $10 \%$. However, as first pointed out in [33], there is an additional uncertainty in the NLL prediction, which is related to the renormalization scheme of the charm-quark mass. In a recent theoretical update of the NLL prediction of the branching ratio of $\bar{B} \rightarrow X_{s} \gamma$, the uncertainty related to the definition of $m_{c}$ was taken into account by varying $m_{c} / m_{b}$ in the conservative range $0.18 \leq m_{c} / m_{b} \leq 0.31$, which covers both the pole mass (with its numerical error) value and the running mass $\bar{m}_{c}\left(\mu_{c}\right)$ value with $\mu_{c} \in\left[m_{c}, m_{b}\right]$; for $E_{\gamma}>1.6 \mathrm{GeV}$ one then finds [34]

$$
\operatorname{BR}\left[\bar{B} \rightarrow X_{s} \gamma\right]=\left(3.52 \pm\left. 0.32\right|_{m_{c} / m_{b}} \pm\left. 0.02\right|_{\mathrm{CKM}} \pm\left. 0.24\right|_{\text {param. }} \pm\left. 0.14\right|_{\text {scale }}\right) \times 10^{-4} \text {. }
$$

The only way to resolve this scheme ambiguity in a satisfactory way is to perform a systematic NNLL calculation, which according to a recent investigation [35] is expected to reduce this uncertainty by a factor 2 . 
Parts of the three principal calculational steps leading to the NNLL result within the effective field theory approach are already done: (a) The full SM theory has to be matched with the effective theory at the scale $\mu=\mu_{W}$, where $\mu_{W}$ denotes a scale of order $m_{W}$ or $m_{t}$. The Wilson coefficients $C_{i}\left(\mu_{W}\right)$ only pick up small QCD corrections, which can be calculated in fixed-order perturbation theory. In the NNLL program, the matching has to be worked out at order $\alpha_{s}^{2}$. The matching calculation to this precision is already finished, including the most difficult piece, the three-loop matching of the operators $\mathcal{O}_{7,8}$ [36]. (b) The evolution of these Wilson coefficients from $\mu=\mu_{W}$ down to $\mu=\mu_{b}$ then has to be performed with the help of the renormalization group, where $\mu_{b}$ is of the order of $m_{b}$. As the matrix elements of the operators evaluated at the low scale $\mu_{b}$ are free of large logarithms, the latter are contained in resummed form in the Wilson coefficients. For the NNLL calculation, this RGE step has to be done using the anomalous-dimension matrix up to order $\alpha_{s}^{3}$. While the three-loop mixing among the four-quark operators $\mathcal{O}_{i}(i=1, \ldots, 6)$ [37] and among the dipole operators $\mathcal{O}_{7,8}$ [38] are already available, the four-loop mixing of the four-quark into the dipole operators is still an open issue. (c) To achieve NNLL precision, the matrix elements $\left\langle X_{s} \gamma\left|\mathcal{O}_{i}\left(\mu_{b}\right)\right| b\right\rangle$ have to be calculated to order $\alpha_{s}^{2}$ precision. This includes also bremsstrahlung corrections. In 2003, the $\left(\alpha_{s}^{2} N_{F}\right)$ corrections to the matrix elements of the operators $\mathcal{O}_{1}, \mathcal{O}_{2}, \mathcal{O}_{7}, \mathcal{O}_{8}$ were calculated [39]. Complete order- $\alpha_{s}^{2}$ results are available to the $\left(\mathcal{O}_{7}, \mathcal{O}_{7}\right)$ contribution to the decay width [40]. Recently, also order $\alpha_{s}^{2}$ terms to the photon energy spectrum (away from the endpoint $E_{\gamma}^{\max }$ ) were worked out for the operator $\mathcal{O}_{7}$ [41].

In this paper we present our calculation of the $O\left(\alpha_{s}^{2}\right)$ contribution to the partonic $b \rightarrow X_{s}^{\text {parton }} \gamma$ decay rate due to the electromagnetic dipole operator $\mathcal{O}_{7}$. Contrary to [40], where this self-interference of the $\mathcal{O}_{7}$ operator was extracted from the imaginary parts of the corresponding 3-loop diagrams via the optical theorem, we calculate the individual cut contributions directly. The advantage of our approach is that it also allows for the calculation of the NNLL interference term of the electromagnetic operator $\mathcal{O}_{7}$ and the chromomagnetic dipole operator $\mathcal{O}_{8}$ and also of the NNLL self-interference term of the operator $\mathcal{O}_{8}$ in a straightforward manner. Applying the optical theorem in a manner analogous to the $\left(\mathcal{O}_{7}, \mathcal{O}_{7}\right)$-interference is not possible in the latter cases, because some cuts contribute to

processes other than the $b \rightarrow X_{s}^{\text {partonic }} \gamma$ decay, and they cannot be separated. We already anticipate here that our results are in complete agreement with the ones presented in [40].

The remainder of this paper is organized as follows. In section 2 we first present our final results, while in section 3 we discuss in some detail the various steps and ingredients of our calculation. Section 4 is reserved for our conclusions.

\section{Results}

Within the low-energy effective theory the partonic $b \rightarrow X_{s} \gamma$ decay rate can be written as

$$
\Gamma\left(b \rightarrow X_{s}^{\mathrm{parton}} \gamma\right)_{E_{\gamma}>E_{0}}=\frac{G_{F}^{2} \alpha_{\mathrm{em}} m_{b}^{2}(\mu) m_{b}^{3}}{32 \pi^{4}}\left|V_{t b} V_{t s}^{*}\right|^{2} \sum_{i, j} C_{i}^{\mathrm{eff}}(\mu) C_{j}^{\mathrm{eff}}(\mu) G_{i j}\left(E_{0}, \mu\right)
$$

where $m_{b}$ and $m_{b}(\mu)$ denote the pole and the running $\overline{\mathrm{MS}}$ mass of the $b$ quark, respectively, $C_{i}^{\text {eff }}(\mu)$ the effective Wilson coefficients at the low energy scale, and $E_{0}$ the energy cut in the photon spectrum. In the present paper, we calculate the function $G_{77}\left(E_{0}, \mu\right)$ for $E_{0}=0$, i.e. 
without a lower cut-off for the photon energy. The function $G_{77}\left(E_{0}, \mu\right)$ corresponds to the self-interference of the electromagnetic dipole operator

$$
\mathcal{O}_{7}=\frac{e}{16 \pi^{2}} m_{b}(\mu)\left(\bar{s} \sigma^{\mu \nu} P_{R} b\right) F_{\mu \nu}
$$

including $\alpha_{s}^{2}$ terms as required for NNLL accuracy. When working to this precision this function can be separated into three different parts,

$$
G_{77}(0, \mu)=\sum_{n=2}^{4} G_{77}^{1 \rightarrow n}(0, \mu)
$$

corresponding to the $n$ particles in the final state, namely the $b \rightarrow s \gamma(n=2), b \rightarrow s \gamma g$ $(n=3), b \rightarrow s \gamma g g$ and $b \rightarrow s \gamma q \bar{q}$ (both $n=4, q \in\{u, d, c, s\})$ transitions. The individual contributions can be written as

$$
\begin{aligned}
G_{77}^{1 \rightarrow 2}(0, \mu) & =1+\frac{\alpha_{s}(\mu)}{4 \pi} X_{21}+\left(\frac{\alpha_{s}(\mu)}{4 \pi}\right)^{2} X_{22}+O\left(\alpha_{s}^{3}\right) \\
G_{77}^{1 \rightarrow 3}(0, \mu)= & \frac{\alpha_{s}(\mu)}{4 \pi} X_{31}+\left(\frac{\alpha_{s}(\mu)}{4 \pi}\right)^{2} X_{32}+O\left(\alpha_{s}^{3}\right) \\
G_{77}^{1 \rightarrow 4}(0, \mu)= & \left(\frac{\alpha_{s}(\mu)}{4 \pi}\right)^{2} X_{42}+O\left(\alpha_{s}^{3}\right),
\end{aligned}
$$

where $\alpha_{s}(\mu)$ is the running coupling constant in the $\overline{\mathrm{MS}}$ scheme. The functions $X_{i j}$ themselves can be naturally divided into two parts,

$$
X_{i j}=X_{i j}^{\mathrm{bare}}+X_{i j}^{\mathrm{ct}}
$$

where the superscripts 'bare' and 'ct' denote the contributions stemming from the unrenormalized amplitudes and from amplitudes involving at least one counterterm $Z$ factor, respectively. Working in the Feynman gauge for the gluon propagator and defining

$$
L_{\mu}=\ln \left(\frac{\mu}{m_{b}}\right)
$$

the contributions of order $\alpha_{s}$ read (inserting the color factors in numerical form)

$$
\begin{aligned}
X_{21}^{\text {bare }} & =-\frac{8}{3 \epsilon^{2}}-\frac{1}{\epsilon}\left(8+\frac{32}{3} L_{\mu}\right)-\frac{64}{3}+\frac{4}{9} \pi^{2}-32 L_{\mu}-\frac{64}{3} L_{\mu}^{2} \\
X_{21}^{\text {ct }} & =-\frac{4}{3 \epsilon}-\frac{20}{3}-\frac{32}{3} L_{\mu} \\
X_{31}^{\text {bare }} & =\frac{8}{3 \epsilon^{2}}+\frac{1}{\epsilon}\left(\frac{28}{3}+\frac{32}{3} L_{\mu}\right)+\frac{316}{9}-\frac{20}{9} \pi^{2}+\frac{112}{3} L_{\mu}+\frac{64}{3} L_{\mu}^{2} \\
X_{31}^{\text {ct }} & =0
\end{aligned}
$$

while those of order $\alpha_{s}^{2}$ are given by

$$
X_{22}^{\text {bare }}=\left(\frac{\mu}{m_{b}}\right)^{6 \epsilon}\left\{\frac{3.55556}{\epsilon^{4}}+\frac{0.444445 N_{L}+1.77778 N_{H}-21.5556}{\epsilon^{3}}\right.
$$




$$
\begin{aligned}
& +\frac{2.96296 N_{L}+4.44444 N_{H}-58.5668}{\epsilon^{2}} \\
& \left.+\frac{15.6452 N_{L}+12.4638 N_{H}-298.294}{\epsilon}+68.4541 N_{L}+20.9389 N_{H}-911.484\right\} \\
& X_{22}^{\mathrm{ct}}=\frac{1}{\epsilon^{3}}\left(\frac{584}{9}-\frac{16}{9} N_{F}\right)+\frac{1}{\epsilon^{2}}\left\{\frac{1762}{9}+\frac{3104}{9} L_{\mu}-\left(\frac{52}{9}+\frac{64}{9} L_{\mu}\right) N_{F}+\frac{4}{3} N_{H}\right\} \\
& +\frac{1}{\epsilon}\left\{\frac{5635}{9}-\frac{196}{27} \pi^{2}+\frac{9172}{9} L_{\mu}+\frac{8512}{9} L_{\mu}^{2}\right. \\
& \left.-\left(\frac{382}{27}-\frac{8}{27} \pi^{2}+\frac{200}{9} L_{\mu}+\frac{128}{9} L_{\mu}^{2}\right) N_{F}+\left(\frac{2}{9}+8 L_{\mu}\right) N_{H}\right\} \\
& +\frac{24403}{18}-\frac{1399}{54} \pi^{2}-\frac{32}{9} \pi^{2} \ln 2-\frac{4912}{27} \zeta_{3}+\left(\frac{28162}{9}-\frac{976}{27} \pi^{2}\right) L_{\mu}+\frac{24316}{9} L_{\mu}^{2} \\
& +\frac{47872}{27} L_{\mu}^{3}+\left(\frac{635}{27}-\frac{41}{9} \pi^{2}+\frac{4}{3} L_{\mu}+24 L_{\mu}^{2}\right) N_{H} \\
& -\left(\frac{583}{27}-\frac{17}{9} \pi^{2}-\frac{128}{27} \zeta_{3}+\left(\frac{1076}{27}-\frac{32}{27} \pi^{2}\right) L_{\mu}+\frac{344}{9} L_{\mu}^{2}+\frac{512}{27} L_{\mu}^{3}\right) N_{F} \\
& X_{32}^{\mathrm{bare}}=\left(\frac{\mu}{m_{b}}\right)^{6 \epsilon}\left\{-\frac{11.1111}{\epsilon^{4}}-\frac{31.1111}{\epsilon^{3}}-\frac{89.9399}{\epsilon^{2}}-\frac{262.232}{\epsilon}-1335.13\right\} \\
& X_{32}^{\mathrm{ct}}=-\frac{1}{\epsilon^{3}}\left(\frac{584}{9}-\frac{16}{9} N_{L}\right)-\frac{1}{\epsilon^{2}}\left\{236+\frac{3104}{9} L_{\mu}-\left(\frac{56}{9}+\frac{64}{9} L_{\mu}\right) N_{L}+\frac{32}{9} L_{\mu} N_{H}\right\} \\
& -\frac{1}{\epsilon}\left\{\frac{28348}{27}-\frac{1364}{27} \pi^{2}+\frac{11344}{9} L_{\mu}+\frac{8512}{9} L_{\mu}^{2}\right. \\
& -\left(\frac{632}{27}-\frac{40}{27} \pi^{2}+\frac{224}{9} L_{\mu}+\frac{128}{9} L_{\mu}^{2}\right) N_{L} \\
& \left.+\left(\frac{4}{27} \pi^{2}+\frac{112}{9} L_{\mu}+\frac{160}{9} L_{\mu}^{2}\right) N_{H}\right\} \\
& -\frac{99004}{27}+\frac{4954}{27} \pi^{2}-\left(\frac{154288}{27}-\frac{7184}{27} \pi^{2}\right) L_{\mu}-\frac{31232}{9} L_{\mu}^{2}-\frac{47872}{27} L_{\mu}^{3} \\
& +\left(\frac{728}{9}-\frac{140}{27} \pi^{2}+\left(\frac{2528}{27}-\frac{160}{27} \pi^{2}\right) L_{\mu}+\frac{448}{9} L_{\mu}^{2}+\frac{512}{27} L_{\mu}^{3}-\frac{512}{27} \zeta_{3}\right) N_{L} \\
& -\left(\frac{14}{27} \pi^{2}+\left(\frac{1264}{27}-\frac{56}{27} \pi^{2}\right) L_{\mu}+\frac{560}{9} L_{\mu}^{2}+\frac{1216}{27} L_{\mu}^{3}-\frac{16}{27} \zeta_{3}\right) N_{H}+\frac{19072}{27} \zeta_{3}
\end{aligned}
$$




$$
\begin{aligned}
X_{42}^{\mathrm{bare}}=\left(\frac{\mu}{m_{b}}\right)^{6 \epsilon}\left\{\frac{7.55556}{\epsilon^{4}}-\frac{0.444444 N_{L}-52.6667}{\epsilon^{3}}-\frac{3.40741 N_{L}-188.729}{\epsilon^{2}}\right. & \\
& \left.\quad-\frac{13.2072 N_{L}-557.390}{\epsilon}-40.3041 N_{L}+1877.18\right\} \\
X_{42}^{\mathrm{ct}}=0 . &
\end{aligned}
$$

These results are valid for $N_{F}$ quark flavors out of which $N_{L}$ are massless, and $N_{H}=N_{F}-N_{L}$ have masses equal to $m_{b}$, i.e. we assume that all quark masses except $m_{b}$ can be neglected. We will address this issue once more at the end of this section.

We note that we have numerically calculated the bare terms of order $\alpha_{s}^{2}$ with Maple [42], requiring ten digits precision. However, when writing the corresponding results in (2.8), we retained only 6 significant digits. ${ }^{2}$.

When summing all up, the poles in $\epsilon$ cancel and we obtain the final result

$$
\begin{aligned}
G_{77}(0, \mu)=1 & +\frac{\alpha_{s}(\mu)}{4 \pi}\left\{\frac{64}{9}-\frac{16}{9} \pi^{2}-\frac{16}{3} L_{\mu}\right\} \\
+ & \left.+\frac{\alpha_{s}(\mu)}{4 \pi}\right)^{2}\left\{\left(3.55556 N_{F}-44.4446\right) L_{\mu}^{2}+\left(21.6168 N_{F}-334.803\right) L_{\mu}\right. \\
& \left.+37.8172 N_{L}-2.16077 N_{H}-519.250\right\},
\end{aligned}
$$

noting that all the 6 significant digits given in this equation are in agreement with the numerical evaluation of the analytical result for $G_{77}(0, \mu)$ given in ref. [40].

We also note that the terms of order $\alpha_{s}^{2} N_{L}$ were calculated some time ago in ref. [39], using a different regularization scheme for infrared and collinear singularities. Taking into account that in [39] the running mass $m_{b}(\mu)$ appearing in (2.1) was expressed in terms of the the pole mass $m_{b}$, we find perfect agreement.

Blokland et al. [40] already investigated in detail the size of the $O\left(\alpha_{s}^{2}\right)$ corrections to the ratio $R=\Gamma\left(b \rightarrow X_{s} \gamma\right) / \Gamma\left(b \rightarrow X_{u} e \bar{\nu}\right)$ and the goodness of the naive non-abelianization hypothesis in various conventions for the factor of $m_{b}$ that normalize the decay rate. As we completely agree with their findings, we do not discuss this issue again.

Instead, we briefly investigate the sensitivity of the order $\alpha_{s}^{2}$ corrections (of course only those related to the $\left(\mathrm{O}_{7}, \mathrm{O}_{7}\right)$ interference) on the charm quark mass. As discussed above, the results in (2.9) are valid for $N_{H}$ flavors with mass $m_{b}$ and for $N_{L}$ massless flavors. As the charm quark mass is somewhere in between, it is interesting to consider the coefficient of $\left(\alpha_{s} /(4 \pi)\right)^{2}$ in (2.9) for the two extreme cases $\left(N_{H}=1, N_{L}=4\right)$ and $\left(N_{H}=2, N_{L}=3\right)$. Denoting this coefficient by $X_{2}\left(N_{H}, N_{L}\right)$, we obtain (for $\mu=m_{b}$ )

$$
X_{2}(1,4)=-370.142 \quad X_{2}(2,3)=-410.120 \text {. }
$$

\footnotetext{
${ }^{2}$ Actually, we also obtained the results for the bare terms proportional to $\alpha_{s}^{2} N_{L}$ in analytical form. However, to get a uniform presentation of the results, we give these terms in numerical form.
} 
The true answer is expected to be somewhere in between. As a consequence the theoretical uncertainty of this coefficient due to the uncalculated $m_{c}$-dependence is about $\pm 5 \%$.

\section{Details of the calculation}

As already discussed in the introduction, we do not calculate the self-interference of the operator $\mathcal{O}_{7}$ by taking the imaginary parts of the corresponding 3-loop diagrams via the optical theorem, as done in [40], but we directly work out the individual two-, three- and four-particle cut-contributions as indicated in (2.4).

In a first subsection we briefly summarize the main practical tools used for the derivation of the unrenormalized contributions to these cuts (denoted by $X_{i j}^{\text {bare }}$ in section 2). In a second subsection we illustrate that we can obtain to very high numerical precision the results for specific diagrams in [40] when summing the contributions of the corresponding cuts. The relevant renormalization constants for the various fields and parameters which are needed to calculate the counterterm contributions can be found in the third subsection.

\subsection{Regularized bare contributions}

The starting point is the general expression for the decay rate of the massive $b$ quark with momentum $p_{b}$ into $2 \leq n \leq 4$ massless final-state particles with momenta $k_{i}$,

$$
\begin{aligned}
\Gamma_{1 \rightarrow n}= & \frac{1}{2 m_{b}}\left(\prod_{i=1}^{n} \int \frac{d^{d-1} k_{i}}{(2 \pi)^{d-1} 2 E_{i}}\right)(2 \pi)^{d} \delta^{(d)}\left(p_{b}-\sum_{i=1}^{n} k_{i}\right)\left|M_{n}\right|^{2} \\
= & \frac{1}{2 m_{b}}(2 \pi)^{n}\left(\prod_{i=1}^{n-1} \int \frac{d^{d} k_{i}}{(2 \pi)^{d}} \delta\left(k_{i}^{2}\right) \theta\left(k_{i}^{0}\right)\right) \\
& \times \delta\left(\left(p_{b}-\sum_{i=1}^{n-1} k_{i}\right)^{2}\right) \theta\left(p_{b}^{0}-\sum_{i=1}^{n-1} k_{i}^{0}\right)\left|M_{n}\right|^{2},
\end{aligned}
$$

where the squared Feynman amplitude $\left|M_{n}\right|^{2}$ is always understood to be summed over final spin-, polarization- and color states, and averaged over the spin directions and colors of the decaying $b$ quark. For $n=4$ it also includes a possible factor of $1 / 2$ if there are two identical particles in the final state. Furthermore, $d=4-2 \epsilon$ denotes the space-time dimension that we use to regulate the ultraviolet, infrared and collinear singularities.

At this point a remark concerning the polarization sums over transverse gluons is in order. There are two well known procedures: a) One takes the full expression for the transversal polarization sum, i.e

$$
\sum_{r=1}^{2} \epsilon_{r \mu} \epsilon_{r \nu}=-g_{\mu \nu}+\frac{t_{\mu} k_{\nu}+k_{\mu} t_{\nu}}{k \cdot t}-\frac{t^{2} k_{\mu} k_{\nu}}{(k \cdot t)^{2}},
$$

where the polarization vectors $\epsilon_{r}$, the momentum $k$ of the gluon, and the arbitrary vector $t$ are chosen such that

$$
\epsilon_{r} \cdot k=0, \quad \epsilon_{r} \cdot \epsilon_{r^{\prime}}=-\delta_{r r^{\prime}}, \quad \epsilon_{r} \cdot t=0, \quad r, r^{\prime}=1,2 .
$$



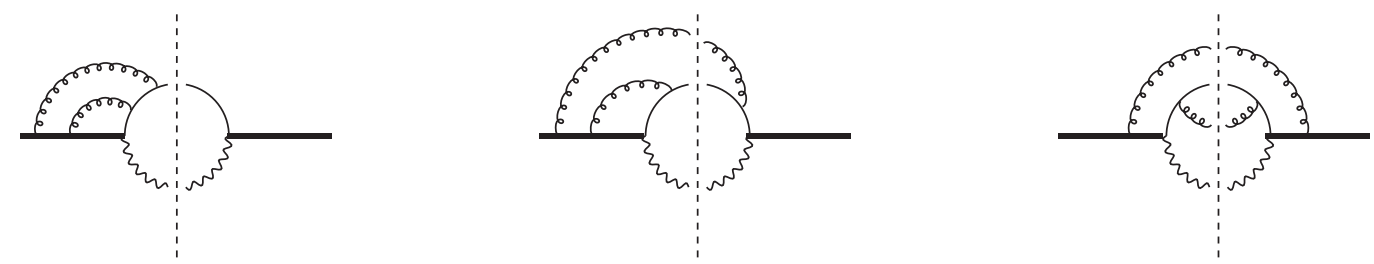

Figure 1: Sample contribution to $\Gamma_{1 \rightarrow n}$ for $n=2$ (left), $n=3$ (center) and $n=4$ (right) at $O\left(\alpha_{s}^{2}\right)$. The vertical line separates the original Feyman diagrams which enter the squared amplitude $\left|M_{n}\right|^{2}$. Thick lines denote the massive $b$ quark, thin lines the massless $s$ quark, wavy lines the photon, and curly lines gluons.

b) Alternatively, one replaces the polarization sum by $-g_{\mu \nu}$ and includes also (unphysical) ghost particles in the final state. We have checked that the two methods give the same results.

The next step concerns the algebraic reduction of the quantities $\Gamma_{1 \rightarrow n}$ being composed of several contributions one of which is depicted in Fig. 1 for the cases $n=2,3$ and 4 at $O\left(\alpha_{s}^{2}\right)$. Following $[43,44]$, we replace the delta functions in the phase-space representation (3.1) by the differences of propagators, e.g.,

$$
\delta\left(q^{2}\right)=\frac{1}{2 \pi i}\left(\frac{1}{q^{2}-i 0}-\frac{1}{q^{2}+i 0}\right),
$$

in order to convert the phase space integrations into loop integrations (which can be combined with possible loop integrations already present in $\left|M_{n}\right|^{2}$ ). In this way the systematic Laporta algorithm [45] based on the integration-by-part technique first proposed in [46, 47] can be applied to reduce scalar products in the numerators and the number of propagators ${ }^{3}$. At $O\left(\alpha_{s}^{2}\right)$ this means that we have to reduce 3-loop diagrams, see Fig. 1 for which we use the AIR implementation of the Laporta algorithm [48] and a self-written one in Mathematica [49].

After the reduction process it usually happens that in some terms the propagators introduced via (3.4) appear with zero or negative power. In this case the $\pm i 0$ prescription becomes irrelevant and as a consequence these terms cancel. In the remaining terms we convert the propagators introduced via (3.4) back to delta-functions.

The final task is thus to perform possible loop integrations contained in $\left|M_{n}\right|^{2}$ together with the phase space integrations. For $n=2$ the phase space integration is trivial,

$$
\Gamma_{1 \rightarrow 2}=\frac{1}{4} \frac{\Gamma\left(\frac{d-2}{2}\right)}{\Gamma(d-2)}(4 \pi)^{1-\frac{d}{2}} m_{b}^{d-5}\left|M_{2}\right|^{2}
$$

and the loop integrations at $O\left(\alpha_{s}^{2}\right)$ can be done numerically by means of the sector decomposition method [50]. The remaining loop integrations at $O\left(\alpha_{s}\right)$ contained in $\left|M_{3}\right|^{2}$ can be

${ }^{3}$ Additional kinematical constraints on the phase space integrations have to be implemented via additional delta-functions in order to be compatible with the integration-by-parts equations [44]. For example the implementation of a lower cut-off $E_{0}$ in the photon energy spectrum can be achieved by inserting the term

$$
\int_{E_{0}}^{\frac{m_{b}}{2}} d E_{\gamma} \delta\left(E_{\gamma}-\frac{p_{b} \cdot p_{\gamma}}{m_{b}}\right)
$$

on the right hand side of (3.1). 
performed via introduction of Feynman parameters as usual. The integration of the latter will also be done numerically by means of the sector decomposition method when combined with an appropriate parametrization of the three-particle phase space, see below. For the treatment of the $n=4$ contributions we follow [51] as far as the phase space representations are concerned (see also [52]). However, as the structure of the matrix elements in our application is slightly different than in [51], certain substitutions of the integration variables are necessary as described below. The (numerical) evaluation of the phase space integrals is then again performed by sector decomposition techniques.

To make the paper self-contained as far as parametrizations of 3- and 4-particle phase spaces are concerned, we look at them in turn. To this end, we define the dimensionless Mandelstam variables

$$
y_{i j \ldots k}=\frac{1}{m_{b}^{2}} s_{i j \ldots k}=\frac{1}{m_{b}^{2}}\left(k_{i}+k_{j}+\cdots+k_{k}\right)^{2} .
$$

For the three-particle process $b \rightarrow s \gamma g$ we obtain

$$
\Gamma_{1 \rightarrow 3}=\frac{1}{4} \frac{(4 \pi)^{1-d}}{\Gamma(d-2)} m_{b}^{2 d-7} \int_{0}^{1} d x_{1} d x_{2}\left(1-x_{1}\right)\left[x_{1}\left(1-x_{1}\right)^{2} x_{2}\left(1-x_{2}\right)\right]^{\frac{d-4}{2}}\left|M_{3}\right|^{2},
$$

where the variables $y_{i j}$ appearing in $\left|M_{3}\right|^{2}$ are understood to be expressed in terms of the two integration variables,

$$
y_{12}=\left(1-x_{1}\right)\left(1-x_{2}\right), \quad y_{23}=\left(1-x_{1}\right) x_{2}, \quad y_{13}=x_{1} .
$$

For the four-particle processes $b \rightarrow s \gamma g g$ and $b \rightarrow s \gamma q \bar{q}$ we take as our starting point (see [51])

$$
\begin{aligned}
\Gamma_{1 \rightarrow 4}=(4 \pi)^{-\frac{3 d}{2}} m_{b}^{3 d-9} \frac{2^{2 d-8} \Gamma\left(\frac{d-2}{2}\right)}{(d-3) \Gamma(d-3)^{2}} \int_{0}^{1} d x_{1} d x_{2} d x_{3} d x_{4} d x_{5}\left[x_{1}\left(1-x_{1}\right)\right]^{d-3} \\
\times x_{2}^{\frac{d-4}{2}}\left(1-x_{2}\right)^{d-3}\left[x_{3}\left(1-x_{3}\right) x_{4}\left(1-x_{4}\right)\right]^{\frac{d-4}{2}}\left[x_{5}\left(1-x_{5}\right)\right]^{\frac{d-5}{2}}\left|M_{4}\right|^{2},
\end{aligned}
$$

where the variables $y_{i j}$ appearing in $\left|M_{4}\right|^{2}$ are understood to be expressed in terms of the five integration variables,

$$
\begin{aligned}
& y_{12}=\left(1-x_{1}\right)\left(1-x_{2}\right)\left(1-x_{3}\right) \\
& y_{13}=\left(y_{13}^{+}-y_{13}^{-}\right) x_{5}+y_{13}^{-} \\
& y_{14}=\left(1-x_{1}\right)\left(x_{2}+x_{3}-x_{2} x_{3}\right)-\left(y_{13}^{+}-y_{13}^{-}\right) x_{5}-y_{13}^{-} \\
& y_{23}=x_{1}\left(1-x_{2}\right) x_{4} \\
& y_{24}=x_{1}\left(1-x_{2}\right)\left(1-x_{4}\right) \\
& y_{34}=x_{1} x_{2} \\
& y_{13}^{ \pm}=\left(1-x_{1}\right)\left[x_{3} x_{4}+x_{2}\left(1-x_{3}\right)\left(1-x_{4}\right) \pm 2 \sqrt{x_{2} x_{3}\left(1-x_{3}\right) x_{4}\left(1-x_{4}\right)}\right] .
\end{aligned}
$$

Of course this is not the only possible parametrization since the pure phase space (3.1) with $\left|M_{4}\right|^{2}=1$ is symmetric with respect to interchanges of the momenta $k_{i}$. Indeed, this symmetry sometimes can be used to avoid the appearance of the square roots $y_{13}^{ \pm}$in the 


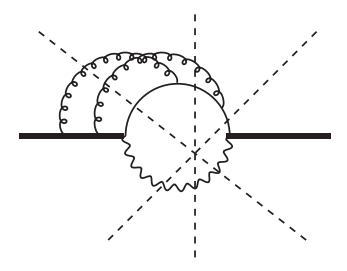

Figure 2: Three-loop diagram (o) of reference [40]. The dashed lines indicate the 2-,3- and 4-particle cuts (see text). Thick lines denote the massive $b$ quark, thin lines the massless $s$ quark, wavy lines the photon, and curly lines gluons.

squared amplitude. We remark that the formulas given above for the three- and the fourparticle phase spaces have the crucial property that the pure phase-space integrals completely factorize when written in these variables.

The expression in (3.9) is a convenient parametrization for the $1 \rightarrow 4$ decay width for the cases where the denominators in $\left|M_{4}\right|^{2}$ do not depend on $y_{13}$ and $y_{14}$. As in [51] one can always remap the momenta of the final state particles in a given diagram such that $y_{14}$ does not occur. In our cases, however, structures of the form $\left(a-y_{13}\right)$ appear in the denominator, where $a$ is a linear combination of some of the remaining $y_{i j}$ such that $\left(a-y_{13}\right)>0$. This is in contrast to the applications considered in [51] where only $y_{13}$ appears in the denominators. For this reason we generalize the expressions given in eqs. (32) and (33) of [51] to our case. Substituting $x_{5}$ in (3.9) by $\hat{x}_{5}$, where

$$
\hat{x}_{5}=\frac{y_{13}\left(x_{5}\right)-y_{13}^{-}}{y_{13}^{+}-y_{13}^{-}} \frac{a-y_{13}^{+}}{a-y_{13}\left(x_{5}\right)},
$$

we obtain the representation

$$
\begin{aligned}
\Gamma_{1 \rightarrow 4}=( & 4 \pi)^{-\frac{3 d}{2}} m_{b}^{3 d-9} \frac{2^{2 d-8} \Gamma\left(\frac{d-2}{2}\right)}{(d-3) \Gamma(d-3)^{2}} \int_{0}^{1} d x_{1} d x_{2} d x_{3} d x_{4} d \hat{x}_{5}\left[x_{1}\left(1-x_{1}\right)\right]^{d-3} \\
& \times x_{2}^{\frac{d-4}{2}}\left(1-x_{2}\right)^{d-3}\left[x_{3}\left(1-x_{3}\right) x_{4}\left(1-x_{4}\right)\right]^{\frac{d-4}{2}}\left[\hat{x}_{5}\left(1-\hat{x}_{5}\right)\right]^{\frac{d-5}{2}}\left(a-y_{13}\left(\hat{x}_{5}\right)\right) \\
& \times\left[\left(a-y_{13}^{+}\right)\left(a-y_{13}^{-}\right)\right]^{\frac{d-5}{2}}\left[\left(1-\hat{x}_{5}\right)\left(y_{13}^{-}-y_{13}^{+}\right)+\left(a-y_{13}^{-}\right)\right]^{4-d}\left|M_{4}\right|^{2},
\end{aligned}
$$

in which the factors of $\left(a-y_{13}\right)$ that appear in the denominators of $\left|M_{4}\right|^{2}$ get cancelled.

\subsection{Illustrative example}

As mentioned, Blokland et al. [40] calculated the order $\alpha_{s}^{2}$ contributions to the decay width by working out (according to the optical theorem) the absorptive part of the $b$-quark selfenergy. At this order the (bare) self-energy is given in terms of the 3-loop diagrams depicted in Fig. 1 of [40]. As an example we consider their diagram (o) and redraw it in Fig. 2. The diagram has a two-, a three- and a four-particle cut as indicated by the straight lines. These cuts represent interferences which are contained in the quantities $\Gamma_{1 \rightarrow n}(n=2,3,4)$ defined above. We denote these interferences by $\Gamma_{1 \rightarrow n}^{(o)}$, where the superscript stands for diagram $(o)$. As we explained in detail in the previous subsection how to calculate such interferences, we 
immediately give the result (putting $\mu=m_{b}$ and working in the Feynman gauge):

$$
\Gamma_{1 \rightarrow n}^{(o)}=\Gamma_{0}\left(\frac{\alpha_{s}^{2}}{4 \pi}\right)^{2} C_{F}\left(C_{F}-\frac{C_{A}}{2}\right)\left(Y_{2}^{(o)}+Y_{3}^{(o)}+Y_{4}^{(o)}\right)
$$

where $\Gamma_{0}$ denotes the decay width at lowest order in QCD. The quantities $Y_{2}^{(o)}, Y_{3}^{(o)}, Y_{4}^{(o)}$, corresponding to the 2- 3- and 4-particle cut, read:

$$
\begin{aligned}
& Y_{2}^{(o)}=0.333333 / \epsilon^{4}+1.66667 / \epsilon^{3}+2.08693 / \epsilon^{2}-6.64830 / \epsilon-67.1138 \\
& Y_{3}^{(o)}=-1.00000 / \epsilon^{4}-5.00000 / \epsilon^{3}-0.970923 / \epsilon^{2}+72.0762 / \epsilon+410.481 \\
& Y_{4}^{(o)}=0.666667 / \epsilon^{4}+3.33333 / \epsilon^{3}-1.11601 / \epsilon^{2}-61.4279 / \epsilon-316.587 .
\end{aligned}
$$

For the sum $Y^{(o)}=Y_{2}^{(o)}+Y_{3}^{(o)}+Y_{4}^{(o)}$ we obtain

$$
Y^{(o)}=4.00000 / \epsilon+26.7798
$$

which is in perfect agreement with the analytic result

$$
Y^{(o)}=4 / \epsilon+\left[\frac{545}{6}-\frac{50}{3} \zeta(3)+\frac{28}{3} \pi^{2} \ln 2-14 \pi^{2}+\frac{14}{45} \pi^{4}\right]=4.00000 / \epsilon+26.7798
$$

of Blokland et al. [40].

\subsection{Renormalization constants}

In this subsection, we collect the relevant renormalization constants necessary to render all bare amplitudes ultraviolet finite and fix the adopted renormalization schemes.

First, the strong coupling constant is renormalized using the $\overline{\mathrm{MS}}$ scheme:

$$
\alpha_{s}^{\text {bare }}=\mu^{2 \epsilon}\left(\frac{e^{\gamma}}{4 \pi}\right)^{\epsilon} Z_{\alpha}^{\overline{\mathrm{MS}}} \alpha_{s}(\mu)
$$

with

$$
Z_{\alpha}^{\overline{\mathrm{MS}}}=1-\frac{33-2 N_{F}}{3 \epsilon} \frac{\alpha_{s}(\mu)}{4 \pi}+O\left(\alpha_{s}^{2}\right) .
$$

Also the $b$ quark mass contained in the operator $O_{7}$ and the Wilson coefficient $C_{7}^{\text {eff }}$ are renormalized in the $\overline{\mathrm{MS}}$ scheme in our calculation. In the present application we only need the product of these two renormalization constants for which we find

$$
Z_{m_{b}}^{\overline{\mathrm{MS}}} Z_{77}^{\overline{\mathrm{MS}}}=1+\frac{4}{3 \epsilon} \frac{\alpha_{s}(\mu)}{4 \pi}-\left(\frac{58-4 N_{F}}{9 \epsilon^{2}}-\frac{543-26 N_{F}}{27 \epsilon}\right)\left(\frac{\alpha_{s}(\mu)}{4 \pi}\right)^{2}+O\left(\alpha_{s}^{3}\right),
$$

in full agreement with $[53,54]$.

All the remaining fields and parameters are renormalized in the on-shell scheme. The on-shell renormalization constant for the $b$ quark mass is given by

$$
Z_{m_{b}}^{\mathrm{OS}}=1-\frac{4}{3} \Gamma(\epsilon) e^{\gamma \epsilon} \frac{3-2 \epsilon}{1-2 \epsilon}\left(\frac{\mu}{m_{b}}\right)^{2 \epsilon} \frac{\alpha_{s}(\mu)}{4 \pi}+O\left(\alpha_{s}^{2}\right) .
$$


For those of the gluon-field and the $s$ quark-field we find

$$
\begin{aligned}
& Z_{3}^{\mathrm{OS}}=1-N_{H} \frac{2}{3} \Gamma(\epsilon) e^{\gamma \epsilon}\left(\frac{\mu}{m_{b}}\right)^{2 \epsilon} \frac{\alpha_{s}(\mu)}{4 \pi}+O\left(\alpha_{s}^{2}\right) \\
& Z_{2 s}^{\mathrm{OS}}=1+N_{H} \frac{4}{3} \frac{\epsilon(1+\epsilon)(3-2 \epsilon) \Gamma(\epsilon)^{2} e^{2 \gamma \epsilon}}{(1-\epsilon)(2-\epsilon)(1+2 \epsilon)(3+2 \epsilon)}\left(\frac{\mu}{m_{b}}\right)^{4 \epsilon}\left(\frac{\alpha_{s}(\mu)}{4 \pi}\right)^{2}+O\left(\alpha_{s}^{3}\right) .
\end{aligned}
$$

Finally, the field renormalization constant of the $b$ quark in the on-shell scheme can be found in the literature [55]:

$$
\begin{aligned}
Z_{2 b}^{\mathrm{OS}}=1-\frac{4}{3} & \Gamma(\epsilon) e^{\gamma \epsilon} \frac{3-2 \epsilon}{1-2 \epsilon}\left(\frac{\mu}{m_{b}}\right)^{2 \epsilon} \frac{\alpha_{s}(\mu)}{4 \pi} \\
+ & \left\{\frac{1}{\epsilon^{2}}\left(30-\frac{4}{3} N_{L}\right)+\frac{1}{\epsilon}\left[\frac{22}{9} N_{L}+\left(\frac{2}{3}+\frac{16}{3} L_{\mu}\right) N_{H}\right.\right. \\
& \left.-\frac{59}{3}+32 L_{\mu}\right]+\left(\frac{113}{9}+\frac{8}{9} \pi^{2}+\frac{152}{9} L_{\mu}+\frac{16}{3} L_{\mu}^{2}\right) N_{L} \\
& +\left(\frac{947}{27}-\frac{10}{3} \pi^{2}+\frac{88}{9} L_{\mu}+16 L_{\mu}^{2}\right) N_{H}-\frac{3383}{18}-\frac{16}{9} \pi^{2} \\
& \left.-\frac{32}{9} \pi^{2} \ln 2+\frac{16}{3} \zeta_{3}-196 L_{\mu}-24 L_{\mu}^{2}\right\}\left(\frac{\alpha_{s}(\mu)}{4 \pi}\right)^{2}+O\left(\alpha_{s}^{3}\right) .
\end{aligned}
$$

\section{Conclusions}

We have presented a calculation of the $O\left(\alpha_{s}^{2}\right)$ contribution associated with the electromagnetic dipole operator $\mathcal{O}_{7}$ to the decay width for $\bar{B} \rightarrow X_{s} \gamma$. In contrast to a previous work [40], the calculational method we used is straightforwardly extendable to the calculation of the interference of the electromagnetic and chromodynamical operator and also to the self-interference of the latter. Our final results are in complete agreement with those in [40]. Additionally, we estimated that the $m_{c}$-dependence of the $O\left(\alpha_{s}^{2}\right)$ contribution of the $\left(\mathcal{O}_{7}, \mathcal{O}_{7}\right)$-interference, which is not worked out in both papers, leads to an uncertainty of about $5 \%$ (of this $O\left(\alpha_{s}^{2}\right)$ contribution).

The NNLL piece calculated in the present paper is an important ingredient of the complete NNLL prediction for the decay rate of the process $\bar{B} \rightarrow X_{s} \gamma$. According to a previous study, it is expected that the theoretical error of the forthcoming NNLL result will be reduced by factor 2 [35]. This increases the high sensitivity of the decay $\bar{B} \rightarrow X_{s} \gamma$ to possible new degrees of freedom even further.

\section{Acknowledgements}

The team from Yerevan is partially supported by the ANSEF N 05-PS-hepth-0/25-338 program. C.G. and T.E. are supported by the Swiss National Foundation; RTN, BBW-Contract No.01.0357 and EC-Contract HPRN-CT-2002-00311 (EURIDICE). 


\section{References}

[1] T. Hurth, Rev. Mod. Phys. 75 (2003) 1159 arXiv:hep-ph/0212304.

[2] S. Bertolini, F. Borzumati and A. Masiero, Phys. Lett. B 192 (1987) 437; S. Bertolini, F. Borzumati, A. Masiero and G. Ridolfi, Nucl. Phys. B 353 (1991) 591.

[3] G. Degrassi, P. Gambino and G. F. Giudice, JHEP 0012, 009 (2000) arXiv:hep-ph/0009337.

[4] M. Carena, D. Garcia, U. Nierste and C. E. M. Wagner, Phys. Lett. B 499, 141 (2001) arXiv:hep-ph/0010003.

[5] G. D'Ambrosio, G. F. Giudice, G. Isidori and A. Strumia, Nucl. Phys. B 645, 155 (2002) arXiv:hep-ph/0207036.

[6] G. Degrassi, P. Gambino and P. Slavich, Phys. Lett. B 635, 335 (2006) arXiv:hep-ph/0601135.

[7] F. Borzumati, C. Greub, T. Hurth and D. Wyler, Phys. Rev. D 62, 075005 (2000) arXiv:hep-ph/9911245.

[8] T. Besmer, C. Greub and T. Hurth, Nucl. Phys. B 609, 359 (2001) arXiv:hep-ph/0105292.

[9] M. Ciuchini, E. Franco, A. Masiero and L. Silvestrini, Phys. Rev. D 67, 075016 (2003) [Erratum-ibid. D 68, 079901 (2003)] arXiv:hep-ph/0212397.

[10] K. Abe et al. [Belle Collaboration], Phys. Lett. B 511 (2001) 151, arXiv:hep-ex/0103042.

[11] S. Chen et al. [CLEO Collaboration], Phys. Rev. Lett. 87 (2001) 251807, arXiv:hep-ex/0108032.

[12] B. Aubert et al. [BABAR Collaboration], Phys. Rev. Lett. 93 (2004) 021804 arXiv:hep-ex/0403035.

[13] B. Aubert et al. [BaBar Collaboration], arXiv:hep-ex/0507001.

[14] P. Koppenburg et al. [Belle Collaboration], Phys. Rev. Lett. 93 (2004) 061803 arXiv:hep-ex/0403004.

[15] Heavy Flavor Averaging Group (HFAG), http://www.slac.stanford.edu/xorg/hfag/rare.

[16] A. G. Akeroyd et al., arXiv:hep-ex/0406071.

[17] J. Hewett et al., arXiv:hep-ph/0503261.

[18] J. Albert et al., arXiv:physics/0512235

[19] K. Adel and Y. Yao, Phys. Rev. D 49 (1994) 4945, arXiv:hep-ph/9308349. 
[20] C. Greub, T. Hurth and D. Wyler, Phys. Lett. B 380 (1996) 385, arXiv:hep-ph/9602281; Phys. Rev. D 54 (1996) 3350, arXiv:hep-ph/9603404.

[21] K. G. Chetyrkin, M. Misiak and M. Munz, Phys. Lett. B 400, 206 (1997) [Erratum-ibid. B 425, 414 (1998)] arXiv:hep-ph/9612313.

[22] C. Greub and T. Hurth, Phys. Rev.D 56 (1997) 2934, arXiv:hep-ph/9703349.

[23] A. J. Buras, A. Czarnecki, M. Misiak and J. Urban, Nucl. Phys. B 611 (2001) 488, arXiv:hep-ph/0105160.

[24] P. Gambino, M. Gorbahn and U. Haisch, Nucl. Phys. B 673, 238 (2003) arXiv:hep-ph/0306079.

[25] A. J. Buras, A. Czarnecki, M. Misiak and J. Urban, Nucl. Phys. B 631 (2002) 219 arXiv:hep-ph/0203135.

[26] H. M. Asatrian, H. H. Asatryan and A. Hovhannisyan, Phys. Lett. B 585, 263 (2004) arXiv:hep-ph/0401038.

[27] A. Ali and C. Greub, Z. Phys. C 49 (1991) 431.

[28] N. Pott, Phys. Rev. D 54 (1996) 938 arXiv:hep-ph/9512252.

[29] A. Czarnecki and W. J. Marciano, Phys. Rev. Lett. 81 (1998) 277 arXiv:hep-ph/9804252.

[30] A. L. Kagan and M. Neubert, Eur. Phys. J. C 7, 5 (1999) arXiv:hep-ph/9805303.

[31] K. Baranowski and M. Misiak, Phys. Lett. B 483, 410 (2000) arXiv:hep-ph/9907427.

[32] P. Gambino and U. Haisch, JHEP 0110, 020 (2001) arXiv:hep-ph/0109058.

[33] P. Gambino and M. Misiak, Nucl. Phys. B 611 (2001) 338 arXiv:hep-ph/0104034.

[34] T. Hurth, E. Lunghi and W. Porod, Nucl. Phys. B 704 (2005) 56 arXiv:hep-ph/0312260.

[35] H. M. Asatrian, C. Greub, A. Hovhannisyan, T. Hurth and V. Poghosyan, Phys. Lett. B 619 (2005) 322 arXiv:hep-ph/0505068 and hep-ph/0512097.

[36] M. Misiak and M. Steinhauser, Nucl. Phys. B 683 (2004) 277 arXiv:hep-ph/0401041.

[37] M. Gorbahn and U. Haisch, Nucl. Phys. B 713 (2005) 291 arXiv:hep-ph/0411071.

[38] M. Gorbahn, U. Haisch and M. Misiak, Phys. Rev. Lett. 95 (2005) 102004 arXiv:hep-ph/0504194.

[39] K. Bieri, C. Greub and M. Steinhauser, Phys. Rev. D 67 (2003) 114019 arXiv:hep-ph/0302051.

[40] I. Blokland, A. Czarnecki, M. Misiak, M. Slusarczyk and F. Tkachov, Phys. Rev. D 72 (2005) 033014 arXiv:hep-ph/0506055. 
[41] K. Melnikov and A. Mitov, Phys. Lett. B 620 (2005) 69 arXiv:hep-ph/0505097.

[42] Maple 9.5, Copyright 2004 by Maplesoft.

[43] C. Anastasiou and K. Melnikov, Nucl. Phys. B 646 (2002) 220 arXiv:hep-ph/0207004.

[44] C. Anastasiou, L. J. Dixon, K. Melnikov and F. Petriello, Phys. Rev. D 69 (2004) 094008 arXiv:hep-ph/0312266.

[45] S. Laporta, Int. J. Mod. Phys. A 15, 5087 (2000) arXiv:hep-ph/0102033.

[46] F. V. Tkachov, Phys. Lett. B 100, 65 (1981).

[47] K. G. Chetyrkin and F. V. Tkachov, Nucl. Phys. B 192, 159 (1981).

[48] C. Anastasiou and A. Lazopoulos, JHEP 0407, (2004) 046 arXiv:hep-ph/0404258.

[49] Mathematica 5.0, Copyright 2003 by Wolfram Research.

[50] T. Binoth and G. Heinrich, Nucl. Phys. B 680, 375 (2004) arXiv:hep-ph/0305234.

[51] C. Anastasiou, K. Melnikov and F. Petriello, Phys. Rev. D 69 (2004) 076010 arXiv:hep-ph/0311311.

[52] A. Gehrmann-De Ridder, T. Gehrmann and G. Heinrich, Nucl. Phys. B682 (2004) 265 arXiv:hep-ph/0311276.

[53] M. Misiak and M. Munz, Phys. Lett. B 344 (1995) 308, arXiv:hep-ph/9409454.

[54] P. Gambino, M. Gorbahn and U. Haisch, Nucl. Phys. B 673 (2003) 238 arXiv:hep-ph/0306079.

[55] K. Melnikov and T. van Ritbergen, Nucl. Phys. B $591 \quad$ (2000) 515 arXiv:hep-ph/0005131. 